\title{
Axial spectral scans of polarization dependent third harmonic generation in a multimode photonic crystal fiber
}

\section{Lorenc \\ lorenc@ilc.sk \\ I. Bugar \\ F. Uherek \\ M. Szpulak \\ W. Urbanczyk}

\author{
International Laser Center, Ilkovicova 3, 81219 Bratislava, Slovakia \\ International Laser Center, Ilkovicova 3, 81219 Bratislava, Slovakia \\ International Laser Center, Ilkovicova 3, 81219 Bratislava, Slovakia
}

Institute of Physics, Wroclaw University of Technology, Wybrzeze Wyspianskiego 27, 50-370 Wroclaw, Poland

Institute of Physics, Wroclaw University of Technology, Wybrzeze Wyspianskiego 27, 50-370 Wroclaw, Poland

Physics Department and International Laser Center, M. V. Lomonosov Moscow State University, Vorob'evy gory, 119992 Moscow, Russia

We demonstrate a nondestructive axial scanning technique for the spectrally resolved analysis of femtosecond nonlinear-optical transformation in photonic crystal fibers. This technique is applied to map the generation of a polarization-switched third harmonic of femtosecond $\mathrm{Cr}$ :forsterite laser pulses in a multimode silica photonic crystal fiber. Obtained results confirmed the intermodal phase-matching to be responsible for the observed polarization dependent multipeak third-harmonic generation. The axial scans revealed, that it is necessary to distinguish between the low and high energy excitation regime of the fiber sample. The proposed technique allows to measure the spectra of nonlinear signals generated in a photonic crystal fiber as a function of the propagation distance without cutting the fiber. [D0I: $10.2971 /$ jeos.2007.07001]

Keywords: Photonic crystal fiber, third harmonic generation, axial scan

\section{Introduction}

Photonic crystal fibers (PCF) [1] are about to accomplish their mission to play substantial role in the field of nonlinear optics. Strong confinement of light in the core region of the PCF and long interaction lengths make it an ideal environment for nonlinear optical phenomena. Because of the attractiveness of supercontinuum generation [2] a considerable interest was paid for broad range of nonlinear effects enhancing the spectral broadening covering self-phase modulation [3], Raman scattering, four-wave mixing [4], second-harmonic generation (SHG) [5], soliton formation and fission [6], soliton selffrequency shift [7]. Recently, efficient third-harmonic generation (THG) was achieved in several cases [8]-[10] using multimode PCFs even for sub-nJ pulse energies. As for any harmonic process, phase-matching condition has to be satisfied in order to achieve efficient TH conversion. Multimode cobweb (high air-fill factor) fibers proved to be a medium suitable for efficient THG [11] due to the intermodal phase-matching. Within the process, one of the lower order modes at the fundamental wavelength is phase-matched to a higher order mode at the third harmonic $(\mathrm{TH})$ requiring the effective index at the fundamental wavelength to be equal to the effective index at the third harmonic: $n_{\text {eff }}^{l}(\omega)=n_{e f f}^{k}(3 \omega), l \neq k$; where $l$ and $k$ denote the mode order. The situation becomes even more challenging for the case of birefringent fiber. Since there is almost always some degree of residual asymmetry of the fiber core as a result of the manufacturing process, nearly all PCF fibers display birefringent nature. Consequently the polarization degeneracy of $n_{\text {eff }}$ dispersion curves is split both for fundamental and higher order modes with the phase-matching condition fulfilled for several pairs of fundamental-third harmonic wavelengths. As a result new components arise in the spectrum of the TH [11].

\section{EXPERIMENTAND MODELING}

A multimode cobweb PCF with an artificial core ellipticity of 1.4:1 and the zero dispersion wavelength close to $910 \mathrm{~nm}$ was employed throughout the experiments (Figure 1). Unamplified femtosecond pulses from a Cr:forsterite oscillator with a central wavelength of $1240 \mathrm{~nm}$ were coupled into the $150 \mathrm{~mm}$ long fiber sample with the typical energy coupled into the core reaching $0.2 n \mathrm{~J}$. The polarization state of incident light was controlled using a combination of a half-wave plate and a polarizer. Farfield mode profiles of the TH modes were obtained by imaging of the blue emission at the output of the fiber on a white screen. An Ocean Optics UV/VIS fiber coupled spectrometer was used in the detection scheme along with the Jobin-Yvon HR640 spectrometer, which covered the NIR region of the spectra. The coupling objective was slightly moved 
in the lateral direction and consequently different modes were excited depending on the position of the lens with respect to the fiber core.

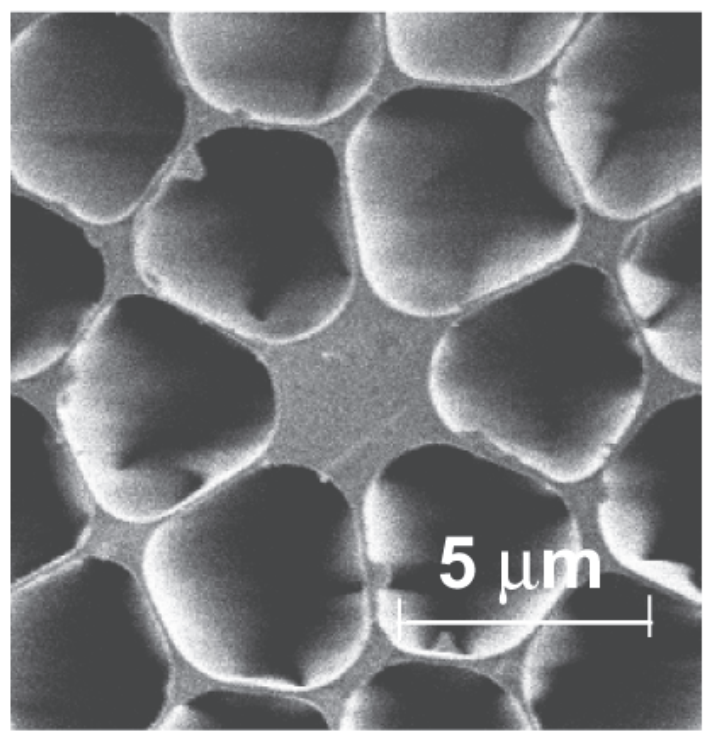

FIG. 1 SEM micrograph showing the facet of the multimode PCF sample with the core ellipticity $1.4: 1$

Two different bright $\mathrm{TH}$ modes with conversion efficiency reaching $0.5 \%$ were observed at the output of the fiber (Figure $2 \mathrm{a}, \mathrm{b}$ ) depending on the coupling geometry. Moreover, rotation of the polarization plane in each of these two cases resulted in mode switching and corresponding changes in the TH spectra (Figure 2c, d).

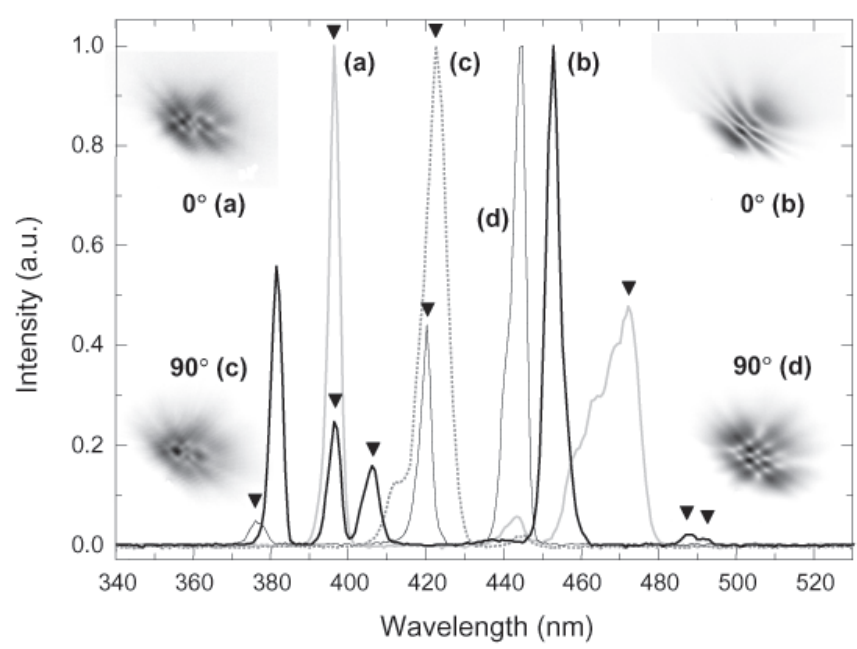

FIG. 2 Observed TH spectra and corresponding farfield mode profiles for polarizations $o(a, b)$ and 90 degrees $(c, d)$ in two different coupling geometries. Theoretically predicted spectral features are marked.

A full-vector mode solver based on a hybrid edge/nodal finite-element method (FEM) [12] was used to calculate the effective refractive indices of the guided modes of the fiber sample. In order to assure the high accuracy of calculations, all simulations were carried out for the real geometry of the fiber. Image processing procedures, like thresholding and bi- narization, applied to SEM image of the fiber cross-section allowed to localize the holes edges and in consequence made it possible to construct the mesh composed of 250000 triangular elements that reproduces real geometry of the analyzed fiber. It was assumed in the calculations that refractive index of silica glass satisfies Sellmeier equation [13]. High order guided modes (up to approximately 50th order) had to be taken into account in order to explain observed spectral features in the short-wavelength part of the spectra. Comparison of the calculated $n_{\text {eff }}$ dispersion behavior (Figure 3) and experimentally obtained TH spectra (Figure 2) shows, that the majority of spectral features might be explained by the intermodal phase-matching scheme.

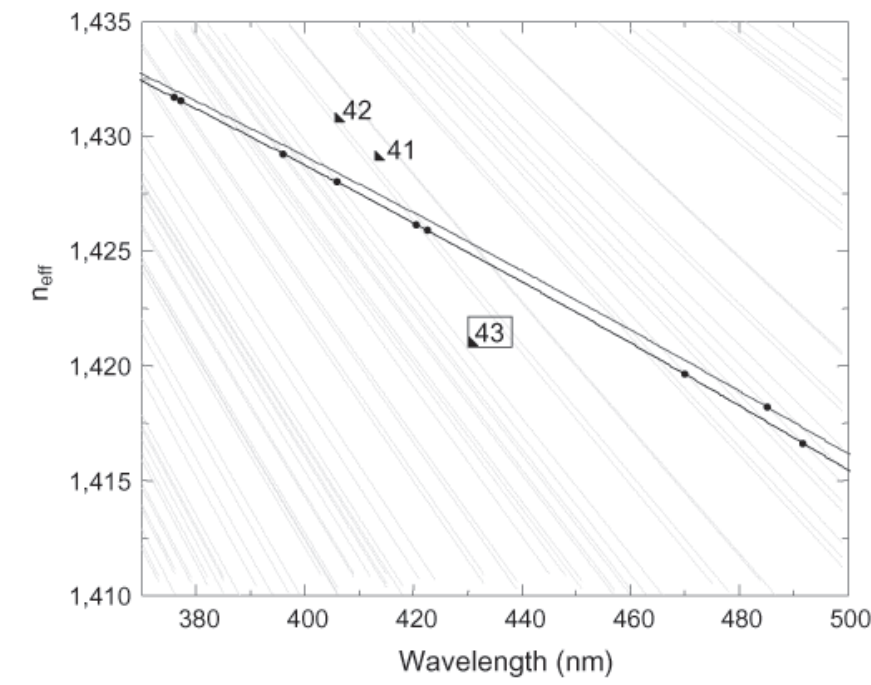

FIG. 3 Calculated phase matching conditions for the intermodal THG inside the PCF sample. The dispersion curves for higher order TH modes (thin lines, numbered) and $3 \omega$ shifted fundamental IR mode (bold lines) are shown.

The calculated dispersion dependence for the polarizationsplit IR fundamental mode is frequency shifted by the three photon process into the TH region where it is overlapped with the higher order modes dispersion curves. Phase-matching is achieved in each intersection point. In a weakly guiding (low air-fill factor) fiber, it is not possible to fulfill the intermodal phase-matching condition due to the small index difference between the core and the cladding. Instead a modified Cherenkov-type [14] phase matching may be achieved. However, Cherenkov circle was not observed in the $\mathrm{TH}$ farfield mode profiles (Figure 2), another argument supporting the intermodal phase-matching scheme. In order to find another evidence for the proposed phase matching scheme, farfield mode profile for the 43rd order guided mode was calculated using a FDTD based mode solver. Result of the simulation shown in Figure 4 corresponding to the 43rd order mode (Figure 3 marked) is compared to the experimental farfield mode profile shown in Figure 2b.

Similar characteristic spatial features could be identified in both cases supporting the model of intermodally phasematched THG. Obviously, not all of the observed spectral features are explained by the three-photon dispersion shift of the fundamental IR mode. In the other cases higher order modes at the fundamental wavelength should be taken into 
account. This concept is supported by the fact that the modified coupling geometry resulted in a completely different set of modes, a behavior similar to what was observed recently in the case of frequency conversion into very high order UV modes [10].

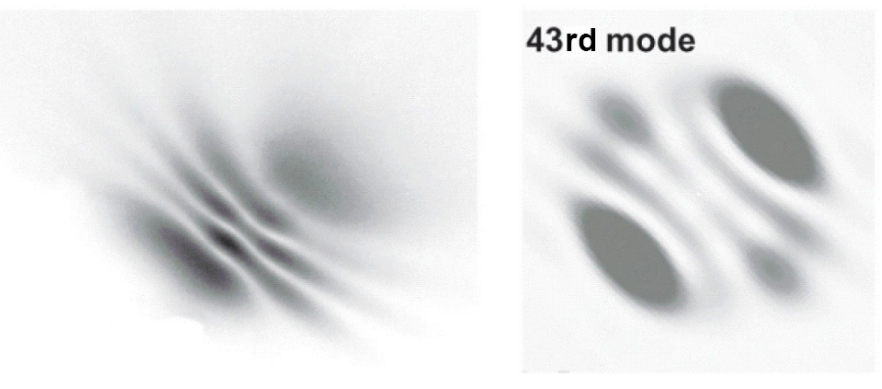

FIG. 4 Experimentally observed (left) and calculated (right) farfield mode profile for the 43 rd order guided TH mode.

\section{AXIAL SPECTRAL SCANS}

Previously [15] a multipeak anharmonic THG was reported using the same sample concluding that the XPM induced phase shift and subsequent peak narrowing might be responsible for the multipeak nature of the TH spectra. This approach also predicts the TH spectral width to evolve as $z^{-1}$ with the propagation coordinate $z$. All predictions of previous work were originally verified by cutting the sample into pieces of different lengths. The method was reliable, yet destructive and time consuming. In order to nondestructively spatially resolve the spectral evolution of the $\mathrm{TH}$ with the ultrashort pulse propagating along the fiber the "axial scan" method was developed within this work. Since there is a substantial Rayleigh scattering in the short-wavelength spectral region, significant amount of light is scattered out of the fiber. Another possible process that could explain the TH light leaving fiber cladding would be the Cherenkov type THG. However, according to the obtained $\mathrm{TH}$ farfield mode profiles, no evidence for such process was found within this work. The scattered light was collected by a fiber tip oriented perpendicularly to the fiber axis. Connected to a fiber coupled spectrometer the tip was scanned axially along the sample and corresponding $\mathrm{TH}$ spectra were recorded in each position. After each scan additional spectra at the fiber output were acquired to check for consistency. The technique allows a high resolution continuous scans to be performed being in contrast to the discrete step data accessible by the conventional fiber-cut approach.

\section{RES ULTS A N D DISCUSSION}

Because of the fiber core anisotropy, both the polarization induced spectral shifts and mode switching occurred when rotating the polarization plane of incident light [16]. In coincidence with observed behavior four axial scans are shown in Figure 5a-d, with incident polarizations corresponding to 0 degrees for the cases a), b) and 90 degrees for the cases c), d).
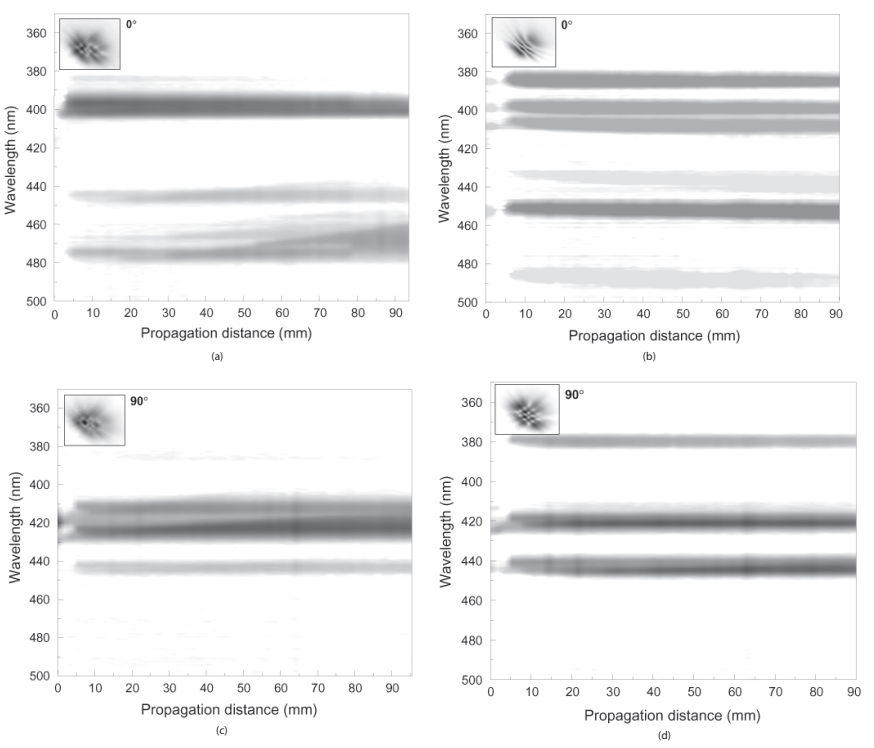

FIG. 5 Axial spectral scans of the TH spectra for polarizations $0(a, b)$ and 90 degrees (c, d) in two different coupling geometries with the corresponding farfield mode profiles given in the insets.

There is virtually no shift of the prominent TH peaks spectral position with the propagation distance [17], although slight broadening occured in the cases depicted on Figure 5a,c. Comparison of Figure 5 with Figure 2 shows, that the spectral information provided by $\mathrm{TH}$ axial scans is consistent with the spectra obtained from the fiber end. Low spectral sensitivity of the detector and the lack of significant Rayleigh scattering at longer wavelengths didn't allow to perform IR axial scans. IR spectra were recorded at the output of the fiber in order to resolve spectral transformations in the region close to the fundamental wavelength (Figure 6).

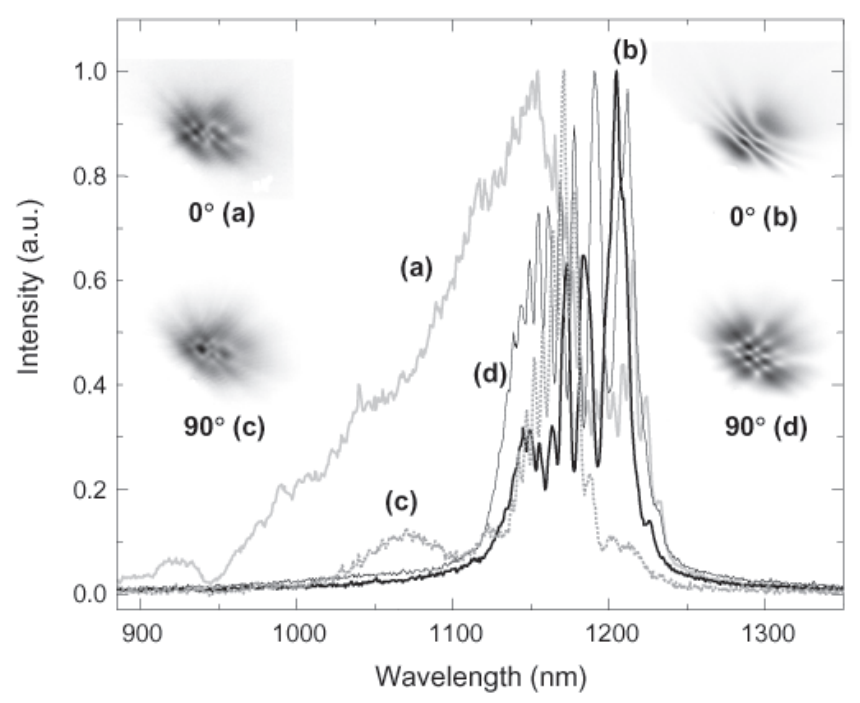

FIG. 6 Observed IR spectra and corresponding farfield TH mode profiles for polarizations $0(\mathrm{a}, \mathrm{b})$ and 90 degrees $(\mathrm{c}, \mathrm{d})$ in two different coupling geometries.

However, spectral range towards the long wavelength side was limited approximately to $1250 \mathrm{~nm}$ due to the spectral sensitivity of the detector. 
Two different regimes related to the broadening of the IR spectra could be identified. In the first coupling geometry (Figure $6 \mathrm{a}, \mathrm{c})$, efficient broadening of the pump spectrum assigned mainly to the self-phase modulation (SPM) and cross-phase modulation (XPM) resulted into spectral widths exceeding $200 \mathrm{~nm}$. Subsequent generation of spectral features in the TH (Figure 2a, c) showed behavior predicted by the SPM/XPM assisted "multimode anharmonic model" (MAM) [15] resulting in broadened $\mathrm{TH}$ features. In the second coupling geometry (Figure 6b,d) less efficient broadening of the pump resulted in the $100 \mathrm{~nm}$ broad IR spectra with multiplets of well resolved peaks observed in the TH (Figure 2b, d). Consequently, less broadening of the IR spectra in the second coupling geometry resulted in narrowband features observed in the $\mathrm{TH}$, a behavior that appears to be due to the limited nonlinear phase change induced by processes such as SPM and XPM. Moreover, within the predictions of the previous model, the TH spectral features are supposed to become narrowband and isolated as the pulse propagates through the fiber. Following the axial scans shown on Figure 5a,c an opposite behavior was found i.e. the TH peaks are broadened during the pulse propagation along the fibre. The spectral width of the $\mathrm{TH}$ peaks didn't change significantly with $z$ in the two remaining cases shown on Figure $5 b$,d. The reason for observed discrepancies with the MAM is the "low energy" regime (unamplified pulses), that doesn't allow the SPM and XPM to account for sufficient nonlinear phase shifts. Indeed, amplified pulses with energies up to $10 \mathrm{~nJ}$ resulted in the TH peak narrowing as reported for the same sample [15] . The most appearing outcome of our work supported by the axial scans is the need to distinguish between two different regimes of THG depending on the pump pulse energies. The obtained results confirmed the developed axial-scan method to be a tool of interest in identification and deeper understanding of different nonlinear phenomena responsible for spectral transformation in PCFs without the need to cut the sample.

\section{C O N CLUSIONS}

Unamplified femtosecond pulses of a Cr:forsterite oscillator induced efficient THG in a multimode birefringent cobweb PCF. Multiplets of peaks in the spectrum of the TH are explained by the intermodal phase matching scheme. Polarization rotation resulted in switching between spectral features allowing to attain control over the composition of the $\mathrm{TH}$ spectra. Novel method of axial spectral scans revealed spectral changes occurring during propagation of the pump pulse through the fiber. According to the obtained results it is necessary to distinguish between the low and high energy regime of the THG inside the sample under study. Axial spectral scans on PCFs proved to be an useful probe into nonlinear spectral transformations showing dependence upon propagation coordinate. Future scope of the method may cover soliton formation and fission, soliton self-frequency shift, XPM, SPM, modulation instabilities and others.

\section{ACK N O W L E D G M N T S}

This work was supported in part by the Ministry of Education grant VEGA No.1/2018/05 and as a part of collaboration within COST P11 action of European Science Foundation.

\section{References}

[1] J. C. Knight,T. A. Birks, P. St. J. Russel and D. M. Atkin, "Allsilica single-mode optical fiber with photonic crystal cladding" Opt. Lett.21, 1547 (1996).

[2] J. K. Ranka, R. S. Windeler, A. J. Stentz, "Visible continuum generation in air-silica microstructure optical fiber with anomalous dispersion at $800 \mathrm{~nm}$," Opt. Lett. 25, 25 (2000).

[3] G. P. Agrawal, Nonlinear Fiber Optics (Academic Press, San Diego, 2001).

[4] S. Coen, A. H. L. Chau, R. Leonhardt, J. D. Harvey, J. C. Knight, W. J. Wadsworth, and P. St. J. Russell, "Supercontinuum generation by stimulated Raman scattering and parametric four-wave mixing in photonic crystal fibers" J. Opt. Soc. Am. B 19, 753 (2002).

[5] J. K. Ranka, R. S. Windeler, A. J. Stentz, "Optical properties of highdelta air-silica microstructure fiber" Opt. Lett. 25, 796 (2000).

[6] J. Herrmann, U. Griebner, N. Zhavoronkov, A. Husakou, D. Nickel, J. C. Knight, W.J. Wadsworth, P. St. J. Russell and G. Korn, "Experimental Evidence for Supercontinuum Generation by Fission of High-Order Solitons in Photonic Fibers" Phys. Rev. Lett. 88, 173901 (2002).

[7] D. T. Reid, I. G. Cormack, W. J. Wadsworth, J. C. Knight and P. St. J. Russell, "Soliton self-frequency shift effects in photonic crystal fibre," J. Mod. Optics 49, 757 (2002).

[8] L. Tartara, I. Christiani, V. Degiorgo, F. Carbone, D. Faccio, M. Romagnoli, W. Belardi, "Phase-matched nonlinear interactions in a holey fiber induced by infrared super-continuum generation" $0 \mathrm{pt}$. Commun. 215, 191 (2003).

[9] F .G. Omenetto, A. Efimov, J. Taylor, J. C. Knight, W. J. Wadsworth, P. St. J. Russell, "Polarization dependent harmonic generation in microstructured fibers" Opt. Express 11, 61 (2003).

[10] A. Efimov, A. J. Taylor, F. G. Omenetto, J. C. Knight, W. J. Wadsworth, P. St. J. Russell, "Nonlinear generation of very high-order UV modes in microstructured fibers" Opt. Express 11, 910 (2003).

[11] A. Efimov, A. J. Taylor, F. G. Omenetto, J. C. Knight, W. J. Wadsworth, P. St. J. Russell, "Phase-matched third harmonic generation in microstructured fibers" Opt. Express 11, 2567 (2003).

[12] M. Koshiba, S. Maruyama and K. Hirayama, "A vector finite element method with the higher order mixed-interpolation-type triangular elements for optical waveguide problems" J. Lightwave Tech. 12, 495 (1994).

[13] R. B. Dyott, Elliptical fiber waveguides, (Artech House Optoelectronics Library 1995).

[14] A. N. Naumov, A. B. Fedotov, A. M. Zheltikov, V. V. Yakovlev, L. A. Me ¿nikov, V. I. Beloglazov, N. B. Skibina, A. V. Scherbakov, "Enhanced $\chi^{(3)}$ interactions of unamplified femtosecond $\mathrm{Cr}$ :forsterite laser pulses in photonic-crystal fibers" J. Opt. Soc. Am. B 19, 2183 (2002).

[15] A. A. Ivanov, D. Lorenc, I. Bugar, F. Uherek, E. E. Serebryannikov, S. 0. Konorov, M. V. Alfimov, D. Chorvat and A. M. Zheltikov, "Multimode anharmonic third-order harmonic generation in a photoniccrystal fiber" Phys. Rev. E 73, 016610 (2006). 
[16] D. Lorenc, I. Bugar, F. Uherek, “Nonlinear spectral shifts in multimode photonic-crystal fibers induced by femtosecond Cr:Forsterite oscillator," in Photonics Crystals and Fibers, W. Urbanczyk, B. Jaskorzynska, P. S. J. Russell, eds., 5950595013 (Proc.
SPIE 2005).

[17] Nevertheless, significant spectral changes occurred in the first few $\mathrm{mm}$ of the fiber (observable on high resolution images) but these will be a subject of separate paper. 